\title{
Resource Limitations, Transmission Costs and Critical Thresholds in Scale-Free Networks
}

\author{
Chung-Yuan Huang \\ Department of Computer Science and \\ Information Engineering \\ Chang Gung University \\ 259 Wen-Hwa 1st Road \\ Taoyuan 333, Taiwan, R.O.C. \\ +886-3-2118800\#3474 \\ gscott@ mail.cgu.edu.tw
}

\author{
Chuen-Tsai Sun \\ Department of Computer Science \\ National Chiao Tung University \\ 1001 Ta Hsueh Road \\ Hsinchu 300, Taiwan, R.O.C. \\ +886-3-571-2121\#56612 \\ ctsun@cs.nctu.du.tw
}

\author{
Chia-Ying Cheng \\ Yu-Shiuan Tsai \\ Department of Computer Science \\ National Chiao Tung University \\ 1001 Ta Hsueh Road \\ Hsinchu 300, Taiwan, R.O.C. \\ +886-3-571-2121\#59284 \\ gis92566@ cis.nctu.du.tw \\ mathoowind@gmail.com
}

\begin{abstract}
Whether or not a critical threshold exists when epidemic diseases are spread in complex networks is a problem attracting attention from researchers in several disciplines. In 2001, Pastor-Satorras and Vespignani used a computational simulations approach to show that epidemic diseases which spread through scale-free social networks do not have positive critical thresholds. In other words, even if a disease has almost no chance of being transmitted from one person to another, it can still spread throughout a scalefree network. However, they ignored two key factors that have a large impact on epidemic dynamics: economic resource limitations and transmission costs. Every infection event entails tangible or intangible costs in terms of time, energy, or money to the carrier, recipient, or both. Here we apply an agent-based modeling and network-oriented computer simulation approach to analyze the influences of resource limitations and transmission costs on epidemic dynamics and critical thresholds in scale-free networks. Our results indicate that when those resources and costs are taken into consideration, the epidemic dynamics of scale-free networks are very similar to those of homogeneous networks, including the presence of significant critical thresholds. It is hoped that our data will help epidemiologists, public health professionals, and computer scientists working with core questions of epidemic diseases, estimates of epidemic dynamics and spreading, and effective public health policies and immunization strategies.
\end{abstract}

\section{Categories and Subject Descriptors}

I.6.3 [Simulation and Modeling]: Applications

\section{General Terms}

Algorithms, Economics, Experimentation, Human Factors.

\section{Keywords}

Critical threshold, economic resource, transmission costs, scalefree networks, effective spreading rate.

Cite as: Resource Limitations, Transmission Costs and Critical Thresholds in Scale-Free Networks, Huang C.Y., Sun C.T., Cheng C.Y., and Tsai Y.S., Proc. of 7th Int. Conf. on Autonomous Agents and Multiagent Systems (AAMAS 2008), Padgham, Parkes, Müller and Parsons (eds.), May, 12-16., 2008, Estoril, Portugal, pp. 1121-1128. Copyright (C) 2008, International Foundation for Autonomous Agents and Multiagent Systems (www.ifaamas.org). All rights reserved.

\section{INTRODUCTION}

Interest in epidemiological issues related to complex network theories has grown considerably ever since Watts and Strogatz [22] proposed their small-world network construction algorithm and Barabási and Albert [2] proposed an improved version of Erdös-Renyi's [4] random networks, which accounts for the power-law connectivity distributions exhibited by scale-free networks $[3,5-7,13,14-18,21]$. Since that time, three global features in social networks and the Internet have been identified and investigated: highly clustered connections in which nodes have many mutual neighbors; the small-world properties of short paths between any two nodes; and long-tailed power-law connectivity distributions in which many nodes have only one or very few connections and a few nodes have a much larger than mean number of connections $[2,12,21]$. Other researchers have noted that the topological features of complex networks exert considerable influence on transmission dynamics and spreading situations associated with epidemic diseases [2, 5-7, 13, 12, 21$22]$, thus allowing for explanations of subtle details of epidemic dynamics that non-network simulation approaches are incapable of producing.

Whether or not a critical threshold exists when epidemic diseases are spread in complex networks is a problem attracting attention from researchers in a range of disciplines [1, 10, 14-18]. The critical threshold is one of the most important indicators of whether or not an epidemic outbreak has occurred. When considering epidemic dynamics, critical thresholds serve as gates for explaining why some epidemic diseases with small numbers of patients at the initial stage evolve into pandemic diseases (e.g., the 1918 influenza), while others disappear shortly after the initial stage. This situation resembles "non-equilibrium phase transitions" (entailing fundamental quantitative and qualitative changes) of concern to epidemiologists. Viable answers to the epidemic/pandemic question can help epidemiologists, public health professionals, and computer scientists to better understand core epidemic disease questions, estimate epidemic dynamics and diffusion situations, and develop effective and public health policies and immunization strategies [3, 14, 18, 21].

Pastor-Satorras and Vespignani report that epidemic diseases which spread within scale-free networks do not have positive critical thresholds. In other words, even if a disease has almost zero chance of being passed from one person to another, it can still be spread within a scale-free network. Researchers who have 
studied epidemic dynamics and critical thresholds in scale-free networks based on Pastor-Satorras and Vespignani's results have consistently concluded that regardless of transmission capability, every infectious disease, computer virus, and cultural trend has a very high probability of being stably transmitted and surviving in a scale-free network $[1,3,8,11,20]$. In short, scale-free networks such as those associated with human sexual contact and the Internet are thought to be capable and vulnerable platforms for spreading epidemic diseases such as HIV and computer viruses.

Pastor-Satorras and Vespignani's [14-18] conclusions are sources of great concern among public health professionals and companies concerned with fighting computer viruses. However, they overlook an important factor-most epidemiological models fail to consider individual differences and resource limitations associated with human interactions and contacts. During transmission processes, every infection event entails tangible or intangible costs in terms of time, energy, or money to the carrier, recipient, or both. In this paper we will refer to all economic resources consumed during the transmission process as transmission costs.

We applied agent-based modeling and network-oriented simulation approach to analyze the influences of resource limitations and transmission costs on epidemic dynamics in scalefree networks. Our results indicate that when those resources and costs are taken into consideration, the epidemic dynamics of scale-free networks are very similar to those of homogeneous networks, including the presence of significant critical thresholds. We believe these results will be useful to epidemiologists and public health professionals working on epidemic dynamics and critical thresholds in scale-free networks, as well as computer and "smart phone" experts concerned with computer virus transmission.

\section{EPIDEMIC DYNAMICS IN COMPLEX NETWORKS}

In a standard epidemiological model, all individuals in a population can be roughly classified into several states, including Susceptible, meaning that an individual is vulnerable to infection but has not yet been infected; Infected, meaning that an individual can infect others; and Removed, meaning that an individual has either recovered, died, or otherwise ceased to pose any further threat. Generally speaking, epidemiologists use combinations of these states to represent orders of transition between different epidemiological phases, giving names such as SIR and SIS to their models.

Past epidemiological diffusion research has focused on the transmission dynamics and outcomes of biologically infectious diseases. A growing number of recent studies are focusing on non-biological and intangible concepts such as computer viruses, cultural influences, rumors, ideas, and beliefs that exist in social networks and on the Internet. In these spreading scenarios, cultural influences move ideas and beliefs between transmitters and receivers, eventually making the majority of receivers behave in the same manner as the transmitters $[6,9,19]$.

In complex networks, nodes are used to represent entities (e.g., organisms or computers) in biological environments or on the Internet. Links indicate close relationships or certain interaction channels between two entities; those with direct connections are called neighbors $[12,22]$. When simulating the transmission dynamics of epidemic diseases in complex networks, epidemiologists usually assume that nodes in complex networks randomly run through an SIS cycle (Susceptible $\rightarrow$ Infected $\rightarrow$ Susceptible). During each time step, each susceptible node is subject to a probability $v$ infection rate if it is connected to one or more infected nodes. Infected nodes recover at a probability $\varepsilon$ recovery rate and once again become susceptible. Based on the definitions of the infection $v$ and recovery $\varepsilon$ rates, effective spreading rate $\lambda$ is defined as $\lambda=v / \delta$. Without a lack of generality, recovery rate $\varepsilon$ can be assigned a value of 1 , since it only affects individuals during a period of disease propagation.

Pastor-Satorras and Vespignani [14] define $\rho(t)$ as the density of infected nodes at time $t$. When time $t$ becomes infinitely large, $\rho$ can be represented as a steady density of infected nodes. Using these definitions, Pastor-Satorras and Vespignani applied dynamic mean-field theory to the SIS model and assumed homogeneous mixing hypothesis according to the topological features of homogeneous networks for obtaining the stable density of infected nodes $\rho$ during long time periods (Eq. 1) as well as the critical threshold $\lambda_{c}$ (Eq. 2).

$$
\begin{aligned}
& \rho=\left\{\begin{array}{cc}
0 & \lambda<\lambda_{c} \\
\frac{\lambda-\lambda_{c}}{\lambda} & \lambda \geq \lambda_{c}
\end{array}\right. \\
& \lambda_{c}=\frac{1}{<k>}
\end{aligned}
$$

According to Equations 1 and 2, a positive and nonzero critical threshold $\lambda_{c}$ exists in a homogeneous network based on the SIS model. If the value of the effective spreading rate exceeds the critical threshold $\left(\lambda \geq \lambda_{c}\right)$, the infection spreads and gains persistence. If the effective spreading rate is below the critical threshold $\left(\lambda<\lambda_{c}\right)$, the infection dies at an exponential speed. In summary, the primary prediction of an SIS epidemiological model in a homogeneous network is the presence of a positive critical threshold, proportional to the inverse of the $\langle k\rangle$ average number of neighbors of every node, below which epidemics die and endemic states are impossible.

Pastor-Satorras and Vespignani relaxed their homogeneity assumption for homogeneous networks and obtained the critical threshold $\lambda_{c}$ in a scale-free network as Equation 3. The results indicate that in scale-free networks with a connectivity exponent of $2<\gamma \leq 3$ and for which $\left\langle k^{2}>\rightarrow \infty\right.$ is the limit of a network of infinite size $(N \rightarrow \infty)$, the critical threshold $\lambda_{c}$ is very close to 0 $\left(\lambda_{c} \rightarrow 0\right)$.

$$
\lambda_{c}=\frac{<k>}{<k^{2}>}
$$

They express the total prevalence $\rho$ for the SIS epidemiological model in a BA scale-free network as a function of the effective spreading rate $\lambda$, and compare it to the theoretical prediction for a homogeneous network. As shown in Figure 1, the total prevalence $\rho$ of a BA scale-free network reaches 0 in a continuous and smooth way when the effective spreading rate $\lambda$ decreases; this indicates an absence of any critical threshold $\left(\lambda_{c}=0\right)$ in a BA 
scale-free network. As long as $\lambda>0$, epidemic diseases can be stably transmitted in the network and eventually reach a steady state. This explains why scale-free networks are fragile in epidemiological spreading situations. Since social networks and the Internet both have "the rich get richer" properties, computer viruses, biologically infectious diseases, and cultural trends can be stably transmitted even when initial infection cases occur in small, limited areas.

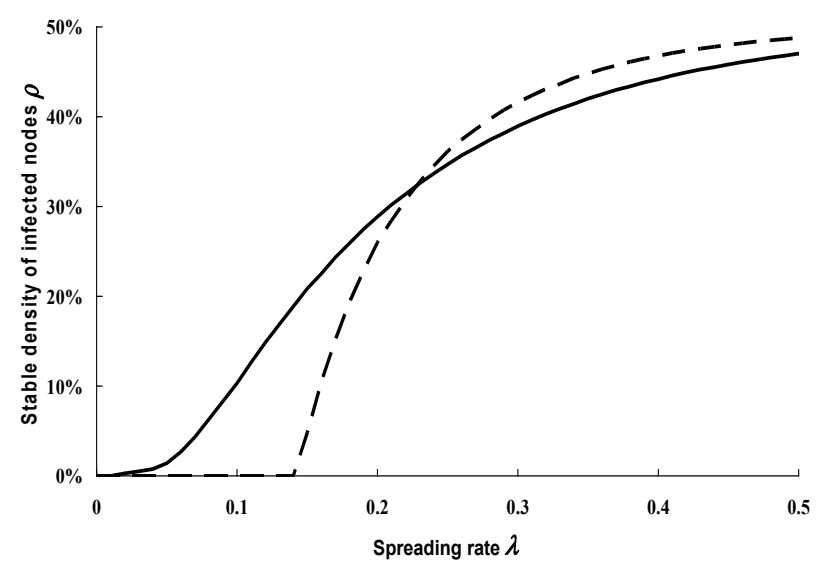

Figure 1. Prevalence $\rho$ in steady state as a function of effective spreading rate $\lambda$. Dashed and solid lines represent BA scalefree and WS small-world networks, respectively.

These conclusions explain the epidemic outbreak mechanisms of biologically infectious diseases and computer viruses. According to traditional epidemiological theory, large-scale pandemics only occur when the effective spreading rate exceeds a specific critical threshold. However, Pastor-Satorras and Vespignani claim that infections can proliferate in scale-free networks regardless of their effective spreading rates. This idea represents major threats to public health concerns and computer data.

For finite-size scale-free networks, Pastor-Satorras and Vespignani [17] introduced the concept of maximum connectivity $k_{c}$ (dependent on $N$ ), which has the effect of restoring a boundary in connectivity fluctuations and inducing an effective nonzero critical threshold. According to the definition of the maximum connectivity $k_{c},<k^{2}>$ in Eq. 3 clearly has a finite value in finitesize scale-free networks. However, in this situation the critical threshold (which is not an intrinsic quantity as it is in homogeneous networks) vanishes as network size increases.

\section{ECONOMIC RESOURCE LIMITATIONS AND TRANSMISSION COSTS}

According to analytical calculations and numerical simulations presented by Pastor-Satorras and Vespignani [14-18], scale-free networks do not have critical thresholds. In other words, they argue that in scale-free networks any infectious disease with a low spreading rate can become an epidemic, which raises serious concerns for epidemiologists, public health professionals, and computer scientists. However, in a world where new infectious diseases, cultural trends, and computer viruses are emerging every day, we know that very few reach epidemic proportions or persist in social networks or the Internet; a majority dies almost immediately following genesis. This situation contradicts Pastor-
Satorras and Vespignani's conclusions, and served as our motivation to look at limitations in transmission processes other than complex network topological features that make it difficult for infectious diseases, trends, or computer viruses to persist.

In addition to the topological characteristics of complex networks, individual differences (e.g., supercarriers, individuals immune to certain infectious diseases) and environmental factors (e.g., mosquito breeding sites) exert considerable influences on transmission dynamics and epidemic disease diffusion. Huang et al. [6] used Watts and Strogatz's small-world networks to investigate the influence of individual differences ("local information") on epidemic simulations. Specifically, they used a sensitivity analysis to show that when an agent-based modeling and network-oriented computer simulation approach is applied to exploring epidemic transmission dynamics in small-world networks, researchers should focus not only on network topological features, but also on proportions of specific values of individual differences related to infection strength or resistance. Less emphasis should be placed on details of the topological connection structures of small-world networks and the distribution patterns of individual difference values.

We believe that many of the studies published in the past five years have been overly focused on ways that the topological features of social networks and the Internet affect epidemic dynamics and critical thresholds. This has occurred at the expense of two key factors: individual differences and economic resources associated with human interactions and contacts. Pastor-Satorras and Vespignani's work on the topological features of scale-free networks is rightfully considered important in terms of epidemiological modeling; their ideas have inspired many studies on critical thresholds and immunization strategies. However, in their analytical calculations and numerical simulation models they assume that transmission events are cost-free. Such an assumption may be suitable for computer viruses that are spread via emails that contain large numbers of recipient addresses, but it is not accurate when applied to infectious diseases or cultural trends.

Economists view resources as tangible or intangible. Available amounts of resources such as time, energy, and money are usually limited. Furthermore, many resources are non-reproducible. Carriers of infectious diseases who spend resources on specific recipients cannot reuse the same resources on other recipients; conversely, recipients cannot reuse resources spent on individual carriers. Using the definition of cost in economics, we will define all resources spent on transmission processes as "transmission costs," and use our agent-based modeling and network-oriented computer simulation approach to (a) prove the appropriateness of introducing the concepts of resource limitations and transmission costs into epidemiological research, and (b) show how doing so exerts a positive impact on public health policies and immunization strategies.

Our task was to find a nonzero, positive critical threshold in a scale-free network under real conditions of economic resource limitations and transmission costs. To this end we applied the state transfer concept of SIS models (Fig. 2) adopted by PastorSatorras and Vespignani [14] as our core simulation model architecture and incorporated parameters to simulate behavioral and transformative results arising from agent interactions. When designing our simulation model we took into consideration the effects of network structure, differences among individuals, 
economic resource limitations, and transmission costs to create a system in which individuals express ranges of behavioral patterns.

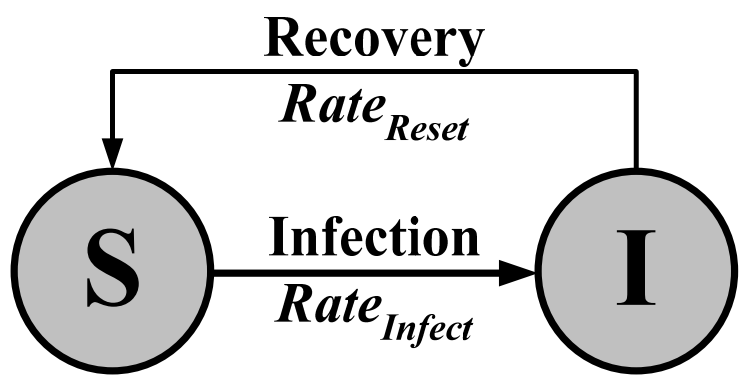

Figure 2. A Susceptible-Infected-Susceptible epidemiological state transfer diagram showing a repetitive cycle.

Each agent (node) in a complex network owns a set of properties and behavioral rules used to demonstrate the features and statuses of persons in social networks or computers connected to the Internet. A link between two nodes means that the connected agents have a close relationship or share a specific interaction/communication channel. An infectious disease or computer virus can be transmitted via this link. At each discrete time step, the epidemiological state of each node is determined by its behavioral rules, original epidemiological state, neighbors' epidemiological states, infection rate, and recovery rate. As stated above, $\rho(t)$ is defined as the density of infected nodes present during time step $t$. When time step $t$ becomes infinitely large, $\rho$ can be represented as a steady density of infected nodes.

A computational flowchart for our proposed simulation model is shown in Figure 3. A complex network $G(N, M)$ with $N$ nodes and $M$ links is constructed using the algorithm described in Section 3 prior to setting relevant parameters and attributes for the nodes involved in the simulation; discrete time $t$ is set at 0 . During a simulation, nodes take turns interacting with neighboring agents for specified time intervals. Note that individual agent interactions do not result in immediate influences and that simultaneous state changes only occur when all agents in a complex network complete their interactions. Accordingly, interaction sequences do not influence interaction processes or results.

At the beginning of each discrete time step, the usable economic resources of each agent $v_{i}$ are reset to $R\left(v_{i}\right)$, meaning that all agents renew and/or receive supplemental resources. For example, for most people their energy level is revived after a night of sleep. In our later experiments, the statistical distribution of individual economic resources can be delta $\left(r_{\text {Constant }}\right)$, uniform, normal, or power-law, as long as the mean value $\langle r\rangle$ satisfies Eq. 4 :

$$
\langle r\rangle=\frac{\sum_{i=1}^{N} R\left(v_{i}\right)}{N}=r_{\text {Constant }}
$$

At each discrete time step, each $v_{j}$ agent chooses and interacts with one neighboring agent from all of its Neighbors $\left(v_{i}\right)$. After the interaction process is finished, agents $v_{i}$ and $v_{j}$ must have transmission costs $c\left(v_{i}\right)$ and $c\left(v_{j}\right)\left(0 \leq c\left(v_{i}\right) \leq R\left(v_{i}\right)\right.$ and $0 \leq c\left(v_{j}\right) \leq$ $R\left(v_{j}\right)$ ) deducted from their respective economic resources regardless of the interaction result. If $R\left(v_{i}\right)<c\left(v_{i}\right)$ after the interaction, agent $v_{i}$ cannot interact with other neighbors because all of its resources have been used up. Otherwise, it repeats the interaction process by choosing another neighboring agent until its resources are exhausted.

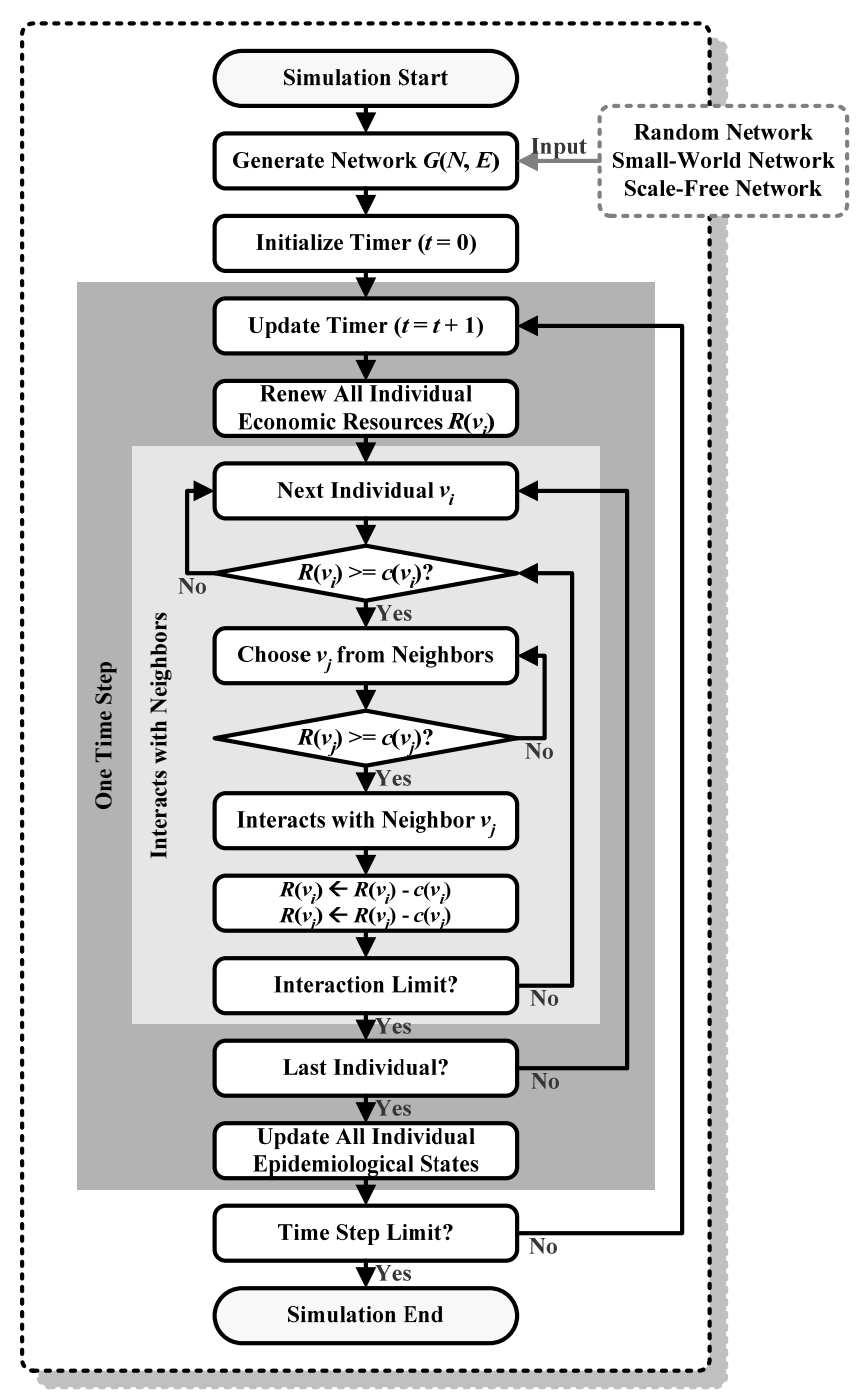

\section{Figure 3. Flowchart for a Susceptible-Infected-Susceptible epidemiological simulation model.}

Assume that infected and contagious agent $v_{i}$ is adjacent to susceptible and infection-prone agent $v_{j}$. When the two agents come into contact, a combination of infection rate Rate $_{\text {Infect }}$, agent $v_{j}$ 's resistance level, and a random number $r$ determines whether or not $v_{j}$ is infected by $v_{i}$. If the random number $r$ is lower than the infection rate Rate $_{\text {Infect }}$, agent $v_{j}$ 's epidemiological state becomes $I$ (Infected). Simultaneously, infected agents are cured and become susceptible with a recovery rate Rate Reset $_{\text {. Without a lack of }}$ generality, recovery rate Rate $_{\text {Reset }}$ can be assigned as 1 , meaning if agent $v_{j}$ is infected by other agents at discrete time step $t-1$, it will recover and become susceptible at discrete time step $t$, since it only takes affect according to the definition of the infection disease propagation time scale.

At the beginning of our simulation of an infectious disease, only ten individuals were given $I$ status; all others were given $S$. During each time step, agents randomly interacted with several 
neighbors. All epidemic experiments discussed in this paper represent average values for 30 runs. Our simulation model is available as a Java application at ftp://anonymous@140.113.88.67; for source code, please contact the authors.

\section{EXPERIMENTAL RESULTS}

We initially used the first simulation experiment to show that an epidemic disease spread within a scale-free social network does have a positive critical threshold if economic resources and transmission costs are taken into consideration - a new conclusion that differs from those reported by previous researchers. The first simulation experiment focused on the universality and generality of the steady density curve and critical threshold when individual economic resources and transmission costs are taken into consideration. Usable economic resources $R\left(v_{i}\right)$ of individual $v_{i}$ at time $t$ was set at 16 units and transmission $\operatorname{cost} c\left(v_{i}\right)$ set at one unit, thus accounting for $6.25 \%$ of the individual's total usable economic resources.

We compared the relationship between effective spreading rate $\lambda$ and steady density $\rho$ for the SIS epidemiological model using three types of complex network platforms: small-world, scale-free without transmission costs, and scale-free with limited individual economic resources and transmission costs. As shown in Figure 4, the eight suites of simulation experiments generated consistent results that did not become contradictory following changes in node and edge numbers. We therefore suggest that the results can be applied to various scale-free networks used to simulate infectious diseases.

The red curves in Figure 4 show that the steady density $\rho$ of the SIS epidemiological model based on scale-free networks reached 0 in a continuous and smooth way when the effective spreading rate $\lambda$ was decreased, indicating the absence of an critical threshold $\left(\lambda_{c}=0\right)$ in scale-free networks without transmission costs. The blue curves show that infectious diseases do have critical thresholds in small-world networks (approximately 0.14). If the value of the effective spreading rate exceeds the critical threshold (i.e., $\lambda \geq \lambda_{c}$ ), the infection will spread throughout the network and eventually reach a steady density $\rho(\lambda)$. If $\lambda<\lambda_{c}$, the infection dies almost immediately. The green curves represent the steady densities $\rho(\lambda)$ of infectious diseases in scale-free networks when individual economic resources and transmission costs are taken into consideration. In addition to being very similar to the blue steady density curves in small-world networks, the green curves have critical thresholds (approximately 0.14). One conclusion drawn from the results of the first simulation experiment is that individual economic resources, transmission costs, and average vertex degrees exert significant influences on epidemic dynamics and critical thresholds in scale-free networks. The same conclusion can be applied to the second and third simulation experiments.

The second simulation experiment was conducted to investigate how the amount of an individual's economic resources $R(v)$ affects the epidemic dynamics and critical thresholds of infectious diseases spread in scale-free networks when the transmission costs are constant. In other words, the second experiment focused on the relationship between the ratio of transmission costs to the total amount of economic resources (hereafter referred to as "the ratio") and critical threshold. To evaluate the influence of the ratio on epidemic dynamics and critical thresholds, we used ten economic resource quantities $(4,8,12,16,20,24,28,32,36$ and 40 units) and assigned the transmission cost $c\left(v_{i}\right)$ of each interaction event as a single unit accounting for $25 \%, 12.5 \%$, $8.33 \%, 6.25 \%, 5 \%, 4.17 \%, 3.57 \%, 3.13 \%, 2.78 \%$ and $2.5 \%$ of an individual's economic resources, respectively.

As shown in Figure 5, the critical threshold significantly increased as the ratio grew. For instance, when the resources $R\left(v_{i}\right)$ of individual $v_{i}$ at time $t$ were designated as 8 units, the critical threshold was approximately 0.22 (Fig. 5, pink curve)significantly greater than that of a small-world network with the same number of nodes and edges (Fig. 4, blue curve) and same average number of vertex degrees (Fig. 4). The opposite was also true: when the $R\left(v_{i}\right)$ of individual $v_{i}$ at time $t$ was designated as 40 units, the shape of the steady density curve (Fig. 5, red curve) was very close to that of the scale-free network without transmission costs (Fig. 5, black curve) and the critical threshold was reduced to 0.09. According to Figure 6, a linear correlation existed between the critical threshold and the ratio. Another interesting observation was that the steady density curve grew at a slower rate as the ratio increased (Fig. 5) - that is, the ratio and steady density had a negative linear correlation. One conclusion drawn from the second simulation experiment is that when transmission costs increase or economic resources decrease, the critical thresholds of spreading infectious diseases in scale-free networks grow, while the steady density shrinks according to diffusion rate.

The third simulation experiment was designed to investigate the effects of economic resource distribution on the epidemic dynamics and critical thresholds of infectious diseases spread in scale-free networks - specifically, to determine how different distribution types (delta, uniform, normal, power-law) of economic resources and their statistical distribution parameters (standard deviation in a normal distribution, number of values and range in a uniform distribution) affect the steady density curves and critical thresholds of infectious disease diffusion in scale-free networks marked by limited individual economic resources and transmission costs.

The orange, green, and purple steady density curves in Figures 7 and 10 represent the delta (fixed value of 16), uniform, and normal distributions of individuals' economic resources, respectively; normal distributions are shown in Figures 8 and 11. We found that all had the same critical threshold $(\approx 0.14)$ and that their steady density curves almost overlapped with each other when the average value of the individuals' economic resources was the same. However, as shown in Figures 9 and 12, if those same economic resources reflected a power-law distribution (i.e., the majority of individuals had extremely limited economic resources and a small number had the most resources) and no correlation existed between the amount of an individual's economic resources and vertex degrees, the resulting steady density curve (pink) grew more slowly than those of the other three distributions, even though they all had the same critical threshold.

The same experimental results were produced as long as the average value of the individuals' economic resources was the same (Figs. 7 and 10). The steady density curves and critical thresholds were almost the same across different distribution types, regardless of whether the individuals' economic resources obeyed a uniform distribution with a range of 2 or 3 (Figs. 8 and 11 , green bars) or a normal distribution with a standard deviation 
of 2 or 3 (Figs. 8 and 11, purple curves). From the two significantly different groups of steady density curves in Figures 7 and 10 (orange, green and purple versus pink), we conclude that as long as researchers ensure that economic resources do not obey a power-law distribution, they can simply assign each individual's $R\left(v_{i}\right)$ at time $t$ as the average value $\langle r>$ (Eq. 11) of the statistical distribution derived from the real-world scenario to facilitate their experiments without affecting simulation results.

\section{CONCLUSION}

Ever since Watts and Strogatz [22] proposed their small-world network model and Barabási and Albert [2] introduced their scalefree network model, epidemiologists, computer scientists, and marketing experts have joined forces to use complex network theories and numerical computer simulations to analyze the details of biological disease and computer virus diffusion. In an attempt to simplify their experiments, researchers have tended to overlook individual economic resources and transmission costsboth of which exert significant impacts on epidemic dynamics. Many have assumed that all transmission events are cost-free and that all individuals have unlimited economic resourcesassumptions that might be applicable to computer viruses spread by the Internet, but not to real-world scenarios such as infectious disease and cultural trend diffusion.

In this paper we used economics definitions for resources and transmission costs, and then proposed three characteristics of economic resources. Two research approaches (agent-oriented modeling and complex networks) were used to construct infectious disease simulation models for the purpose of investigating how economic resources and transmission costs influence epidemic dynamics and thresholds in scale-free networks. Our results indicate that when economic resources and transmission costs are taken into consideration, a critical threshold does in fact exist when infectious diseases are spread within a scale-free network.

According to the results of our first set of experiments, individual economic resources, transmission costs, and average vertex degrees are among factors exerting significant impacts on critical thresholds; node and edge numbers were found to have little impact. The second experimental set provided insight into how the ratio of single transmission event cost to total amount of an individual's economic resources affects stable density curves and critical thresholds. When transmission costs increase or when the total amount of an individual's economic resources decrease, the critical threshold of an infectious disease in a scale-free network grows and stable density is reduced under certain transmission rates. The third set of experiments proved that regardless of whether the individuals' economic resources obey a delta, uniform, or normal distribution, they all have the same stable density curve and critical threshold as long as the average value remains the same across different networks. This conclusion can make the process of constructing simple and abstract computer models significantly less complex.

We suggest that our three main conclusions can help epidemiologists and public health professionals understand core questions of disease epidemics, predict epidemic dynamics and diffusion, and develop effective public health policies and immunization strategies. They may also have utility for computer scientists wanting to develop strategies against infectious viruses at various levels of intrusion as well as computer network viruses.

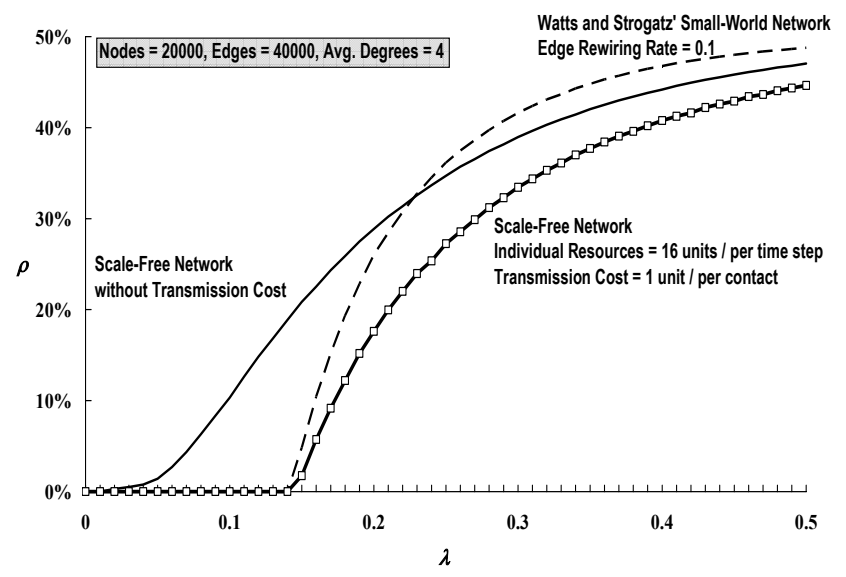

Figure 4. Relationship between effective spreading rate and steady density of the SIS epidemiological model on three types of complex network platforms.

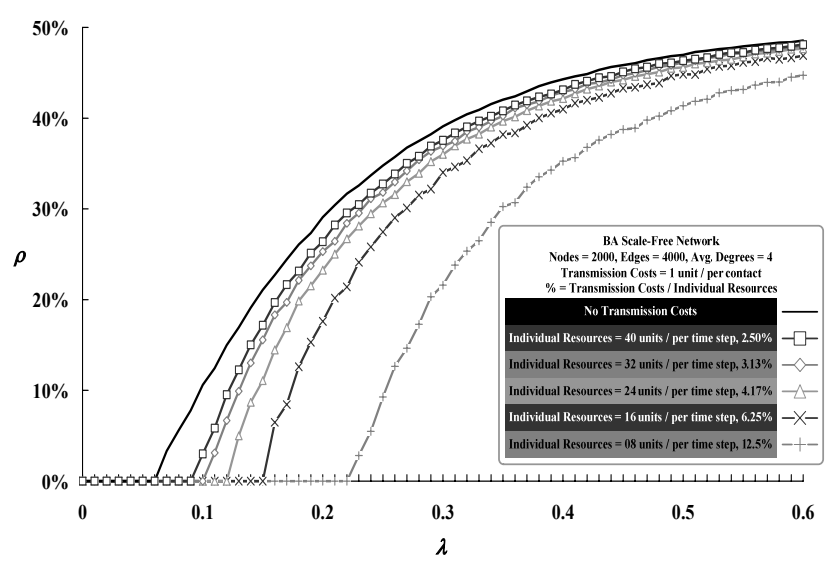

Figure 5. How the amount of an individual's economic resources affect steady density curves.

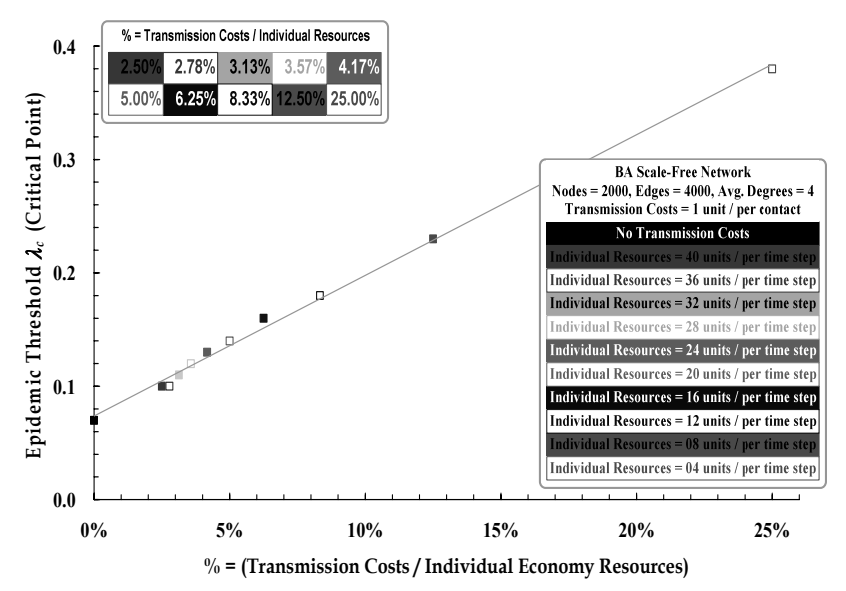

Figure 6. Relationship between ratio of transmission costs to an individual's economic resources and critical threshold. 


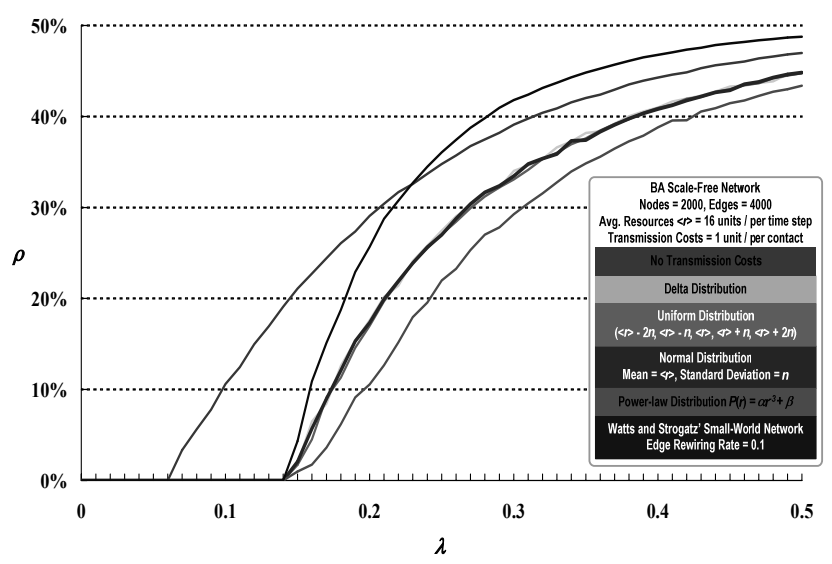

Figure 7. How different distribution types of individual economic resources (delta, uniform, normal, power-law) affect steady density curves and critical thresholds of infectious disease diffusion in a scale-free network.

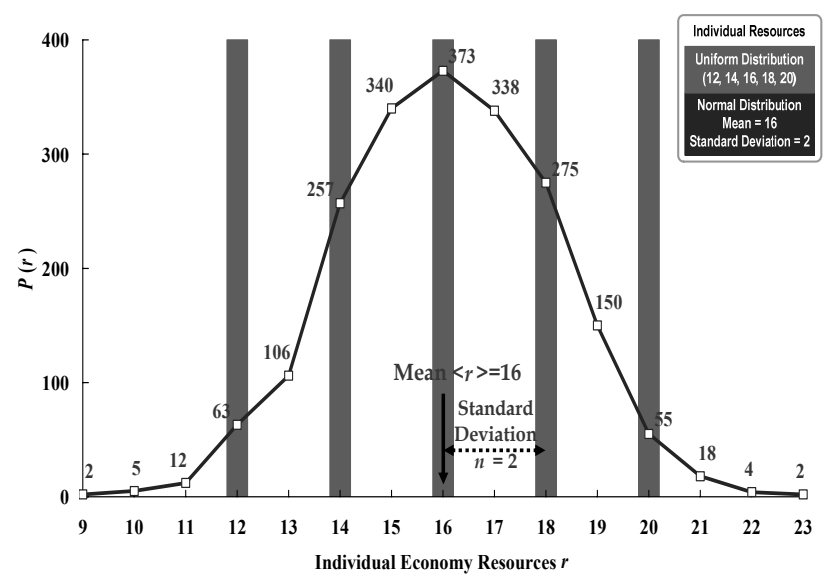

Figure 8. A uniform $(n=5, r=2)$ and normal distribution (standard deviation $=2$ ) of individual economic resources with average value $<r>$ of 16 .

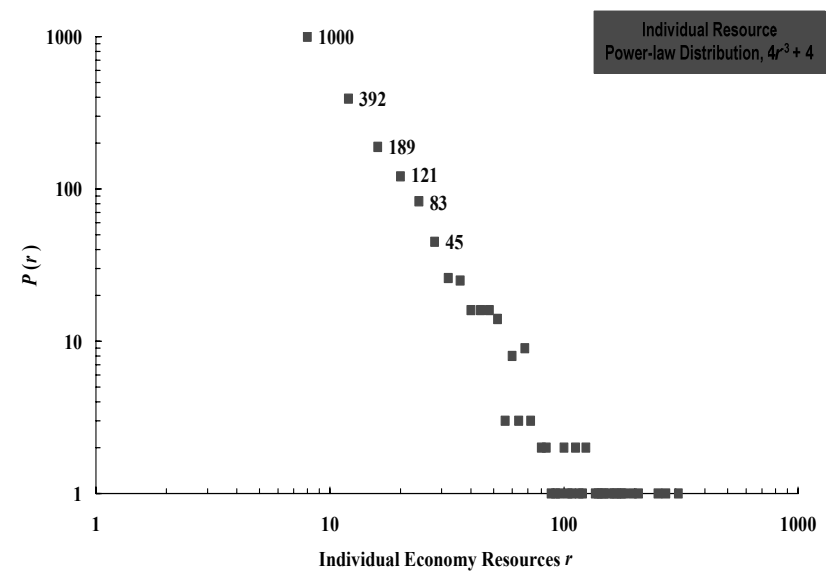

Figure 9. Individual economic resources in a power-law distribution.

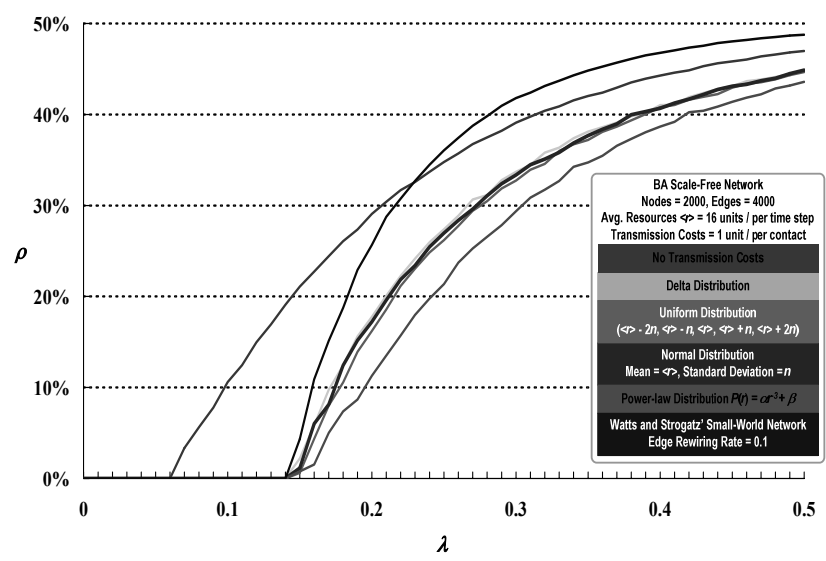

Figure 10. How different types of individual economic resource distributions (delta, uniform, normal, and power-law) affect steady density curves and critical thresholds of infectious disease diffusion in a scale-free network.

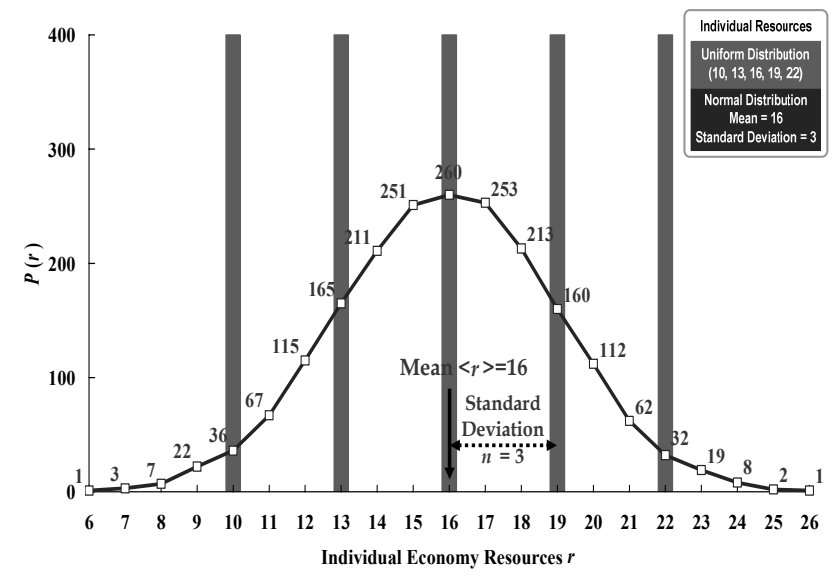

Figure 11. A uniform $(n=5, r=3)$ and normal distribution (standard deviation $=3$ ) of individual economic resources with average value $<r>$ of 16 .

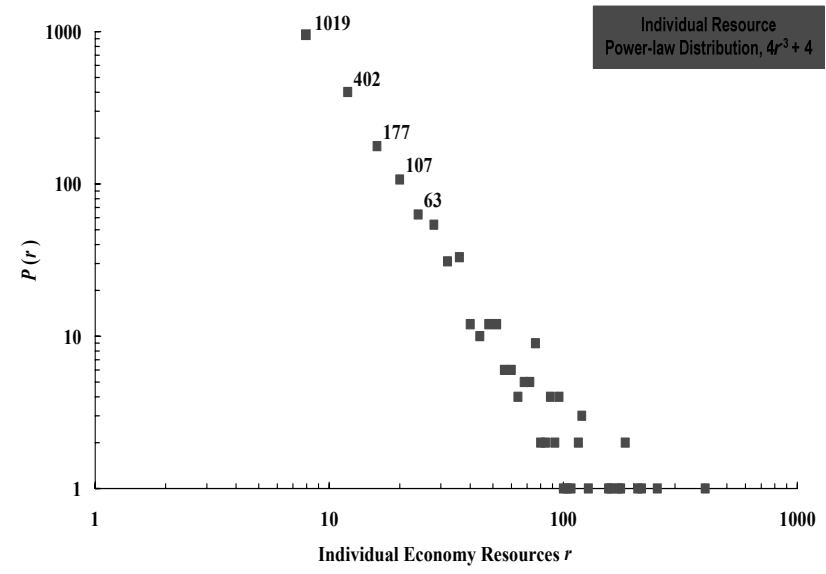

Figure 12. Individual economic resources in a power-law distribution. 


\section{ACKNOWLEDGMENTS}

This work was supported in part by National Science Council, Taiwan, Republic of China under grant NSC 96-2221-E-182-041 and in part by Chang Gung Memorial Hospital under Grants CMRPD260021.

\section{REFERENCES}

[1] Boguñá, M. and Pastor-Satorras, R. 2002. Epidemic spreading in correlated complex networks. Physical Review $E, 66,047104$.

[2] Barabási, A. L. and Albert, R. 1999. Emergence of scaling in random networks. Science, 286(5439), 509-512.

[3] Dezsö, Z. and Barabási, A. L. 2002. Halting viruses in scalefree networks. Physical Review E, 65, 055103.

[4] Erdös, P. and Renyi, A. 1959. On the evolution of random graphs. Publication of the Mathematical Institute of the Hungarian Academy of Science, 5, 17-60.

[5] Huang, C. Y., Sun, C. T., Hsieh, J. L. and Lin, H. 2004. Simulating SARS: Small-world epidemiological modeling and public health policy assessments. Journal of Artificial Societies and Social Simulation, 7(4), http://jasss.soc.surrey.ac.uk/7/4/2.html.

[6] Huang, C. Y., Sun, C. T. and Lin, H. C. 2005. Influence of local information on social simulations in small-world network models. Journal of Artificial Societies and Social Simulation, 8(4), http://jasss.soc.surrey.ac.uk/8/4/8.html.

[7] Huang, C. Y., Sun, C. T., Hsieh, J. L., Chen, Y. M. A. and Lin, H. 2005. A novel small-world model: Using social mirror identities for epidemic simulations. Simulation: Transactions of the Society for Modeling and Simulation International, 81(10), 671-699.

[8] Liu, Z. H. , Lai, Y. C. and Ye, N. 2004. Propagation and immunization of infection on general networks with both homogeneous and heterogeneous components. Physical Review E, 67, 031911.
[9] Lynch, A. 1998. Thought Contagion: How Belief Spreads through Society. New York: Basic Books.

[10] May, R. M. and Lloyd, A. L. 2001. Infection dynamics on scale-free networks. Physical Review E, 64, 066112.

[11] Moreno, Y., Gómez, J. B. and Pacheco, A. F. 2003. Epidemic incidence in correlated complex networks. Physical Review E, 68, 035103.

[12] Newman, M. E. J. 2003. The structure and function of complex networks. SIAM Review, 45, 167-256.

[13] Newman, M. E. J. and Watts, D. J. 1999. Scaling and percolation in the small-world network model. Physical Review E, 60, 7332-7342.

[14] Pastor-Satorras, R. and Vespignani, A. 2001. Immunization of complex networks. Physical Review E, 65, 036134.

[15] Pastor-Satorras, R. and Vespignani, A. 2001. Epidemic spreading in scale-free networks. Physical Review Letter, 86(4), 3200-3203.

[16] Pastor-Satorras, R. and Vespignani, A. 2001. Epidemic dynamics and endemic states in complex networks. Physical Review E, 63, 066117.

[17] Pastor-Satorras, R. and Vespignani. A. 2002. Epidemic spreading in finite size scale-free networks. Physical Review $E, 65,035108$.

[18] Pastor-Satorras, R. and Vespignani, A. 2003. Epidemics and immunization in scale-free networks. In Bornholdt $\mathrm{S}$ and Schuster H G (eds.), Handbook of Graphs and Networks, Wiley-VCH.

[19] ROGERS E M (2006) Diffusion of Innovations (5th ed.). New York: The Free Press.

[20] Volchenkov, D., Volchenkova, L. and Blanchard, P. 2002. Epidemic spreading in a variety of scale free networks. Physical Review E, 66: 046137.

[21] Watts, D. J. 2003. Six Degrees: The Science of a Connected Age. New York: W. W. Norton \& Company.

[22] Watts, D. J. and Strogatz, S. H. 1998. Collective dynamics of 'small-world' networks. Nature, 393(6684), 440-442. 